\title{
Interface control mechanisms in horizontal zone-melting with slow rotation
}

\author{
C.W. Lan*, J.H. Chian, T.Y. Wang \\ Department of Chemical Engineering, National Taiwan University, Taipei 10617, Taiwan, ROC \\ Received 22 February 2000; accepted 25 May 2000 \\ Communicated by D.T.J. Hurle
}

\begin{abstract}
Using slow rotation to control the interfaces in horizontal zone-melting (HZM) is a widely adopted method for organic materials, i.e., the Pfann-Miller technique. A short and nearly axisymmetric zone can be achieved easily even with a rotation rate of only 1 or $2 \mathrm{rpm}$. However, the melt convection might not be the crucial mechanism for such a control. Through flow and interface visualization during HZM of succinonitrile, the role of the slow rotation was investigated. Three-dimensional numerical simulation was further performed to illustrate the key mechanisms for determining the zone shape. Besides the good agreement between the simulation and the experiments, we found that the heat of fusion released or absorbed during the rotational regrowth and remelting played a crucial role, and it could not be ignored in the simulation. (C) 2000 Elsevier Science B.V. All rights reserved.
\end{abstract}

PACS: $44.25 .+\mathrm{f} ;$ 47.27.Te; 81.10.Fq; 02.60.Cb; 02.70.Fj

Keywords: Zone-melting; Interfaces; Rotation; Convection; Stefan problem

\section{Introduction}

The control of the zone shape and convection in zone melting is an important task for both crystal growth and zone refining. The horizontal configuration for zone melting is particularly popular due to its better mixing and interface control [1]. Pfann et al. [1,2] first demonstrated the effectiveness of the slow ampoule rotation on the zone shape control in the horizontal zone-melting (HZM), the so-called Pfann-Miller technique or the rotation-

\footnotetext{
* Corresponding author. Tel./fax: + 886-2-23633917.

E-mail address: lan@ruby.che.ntu.edu.tw (C.W. Lan).
}

convection heating. With only $2.5 \mathrm{rpm}$ rotation, a nearly axisymmetric zone, as short as $20 \%$ of the diameter, was found possible for resorcinol. Nearly flat interfaces could be controlled as well. Since then, this Pfann-Miller technique has been used routinely in the zone refining of organic and inorganic materials. Another way that can also produce an axisymmetric zone is by "high speed" rotation as proposed by Wilcox et al. [3]. The effectiveness of the slow rotation on the zone shape control has been believed to be the cause of melt convection, which behaves like a cutting blade to smooth out the interfaces $[1,2]$. As pointed out by Pfann et al. [2], to make the technique work, the flow pattern due to natural convection cannot be significantly 
altered, which is in contrast to the high-speed rotation [3]. However, the detailed coupling mechanisms of the convection and interfaces during slow rotation have not been discussed. To provide a clearer picture on the interface control mechanisms, theoretical investigation through computer simulation will be helpful. Unfortunately, no theoretical work so far has been devoted to this subject. Indeed, the heat flow and zone shape calculations for HZM require a three-dimensional (3D) model, and of course the simulation is not trivial. Especially, the coupling of the interfaces with the convection is significant due to the large Prandtl number. Furthermore, as the rotation is imposed, the rotational remelting and regrowth needs to be considered at the interfaces. To the best of our knowledge, this kind of Stefan problem has not yet been investigated so far for the HZM crystal growth or refining.

In this report, we performed first the flow and zone shape visualizations during HZM of succinonitrile (SCN). Its 3D simulation including the interface shapes was then conducted. Through the numerical simulation, the mechanisms for the zone shape control were discussed. A simplified configuration as a benchmark problem for the comparison with a commercial code, Fluent [4], was also considered to support our 3D calculations and to better illustrate the role of slow rotation on the heat flows. In the following section, experimental and numerical methods are described briefly. Results and discussion are presented in Section 3, followed by conclusions in Section 4.

\section{Visualization experiments and numerical modeling}

The schematic representation of the experimental setup is shown in Fig. 1. Due to the more reliable thermal properties [5], SCN was chosen as a model material in this study. The raw material (99\% purity) was zone refined ten times to achieve a melting point of nearly $58^{\circ} \mathrm{C}$, which was very close to the reported value for $7 \mathrm{~N} \mathrm{SCN} \mathrm{[6].} \mathrm{With}$ such a high purity, transparent single crystals could be easily grown with a substantially high growth rate (up to several centimeters per hour). The am-

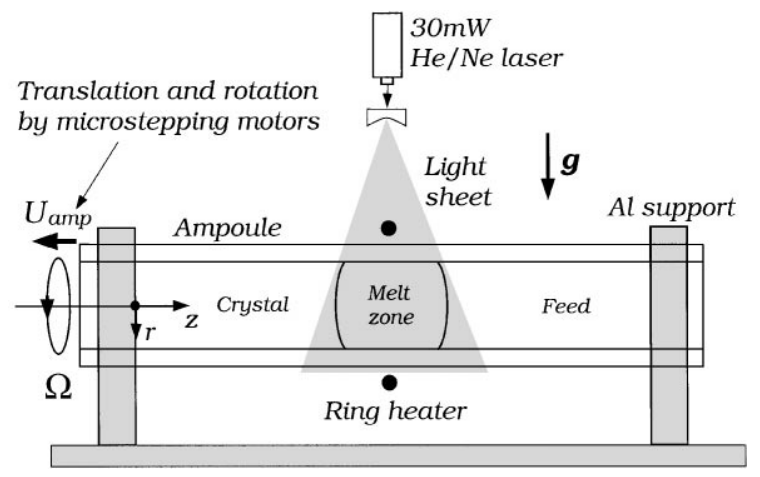

Fig. 1. Schematic representation of the experimental setup for horizontal zone-melting (HZM) of SCN and its flow visualization.

poule was made of a Pyrex tube (ID $=0.9 \mathrm{~cm}$, $\mathrm{OD}=1.1 \mathrm{~cm}$, and length $=30 \mathrm{~cm}$ ). For forming the melt zone, a simple ring heater made of a Nichrome wire $(0.5 \mathrm{~mm}$ in diameter) was further used. Two kinds of heaters were used; one nearly contacted with the ampoule and the other had about a 2-mm gap from the ampoule wall. Interestingly, the observed flow patterns and the zone shape were not changed much by the heating configurations as long as the zone size was kept the same. To observe the convection, a small amount of aluminum powder $(30 \mu \mathrm{m})$ was added in advance to SCN and then visualized (light-cut) by a $30-\mathrm{mW} \mathrm{He} / \mathrm{Ne}$ laser. Another tracer (liquid-crystal capsule powder) with a specific weight closer to SCN was also used, but the picture quality was much worse. Since the observed flow patterns by these two powders were about the same, the aluminum powder was still used. To better observe the interface shapes, a single crystal was grown before zone melting. Furthermore, in each experiment, the stable zone needed to be formed by increasing the heating power to avoid the bubble formation due to zone shrinking (the melt density is lower than the solid). Both translation and rotation were controlled by microstepping motors.

The numerical model used here was similar to that presented in the previous report [7], which included the numerical solution of the Navier-Stokes and heat transfer equations. The most important boundary condition, or the socalled Stefan equation, is at the interfaces for the 
energy balance:

$Q_{\mathrm{m}}-Q_{\mathrm{s}}+\gamma \mathrm{St}\left[\left(\boldsymbol{v}_{z}-\frac{\partial h_{\mathrm{i}}}{\partial \tau}\right) \boldsymbol{e}_{z} \cdot \boldsymbol{n}+r \Omega \boldsymbol{e}_{\phi} \cdot \boldsymbol{n}\right]=0$,

where $Q_{\mathrm{m}}$ and $Q_{\mathrm{s}}$ are the dimensionless heat transfer fluxes (including both convection and conduction) at the melt and solid sides, respectively; $i=\mathrm{f}$ for the feed and $i=\mathrm{c}$ for the crystal. Also, $\gamma$ is the density ratio of the solid to the melt. The Stefan number $\mathrm{St} \equiv \Delta H / C p_{\mathrm{m}} T_{\mathrm{m}}$ scales the heat of fusion with the sensible heat in the melt. The larger the Stefan number, the more important the heat of fusion is. $v_{z}$ is the axial velocity at the interfaces, $h_{\mathrm{i}}$ the interface position, and $\tau$ the dimensionless time. Also, $\boldsymbol{e}_{z}$ and $\boldsymbol{e}_{\phi}$ are the unit vectors, respectively, in the axial and the azimuthal angle direction, while $\boldsymbol{n}$ is the unit normal vector at the interfaces pointing to the melt. $r \Omega \boldsymbol{e}_{\phi}$ is the angular velocity, i.e., $v_{\phi} \boldsymbol{e}_{\phi}$, at the interfaces, where $r$ is the dimensionless radial distance and $\Omega$ the dimensionless angular rotation rate. As a steady state is achieved, $\partial h_{\mathrm{i}} / \partial \tau=0$ and $v_{z}=0$ for no translation. Clearly, if the interfaces are not axisymmetric, continuous solidification (at which $\boldsymbol{n} \cdot \boldsymbol{e}_{\phi}<0$ ) and melting $\left(\boldsymbol{n} \cdot \boldsymbol{e}_{\phi}>0\right)$ will proceed with rotation $(\Omega>0)$.

The heat transfer between the ampoule and the heater was described by a simple convective heat transfer equation [7], where the effective ambient temperature was assumed to be a Gaussian distribution. The peak heater temperature used in the simulation was determined by the thermal measurements at the ampoule surface. The heat transfer coefficient was also used to fit the zone size for the case without rotation. With $1 \mathrm{rpm}$ ampoule rotation, the same heat transfer coefficient was used. Again, as long as the zone size was kept about the same, the interface shapes and the flow structures were not changed much by the thermal profiles, which was also consistent with experimental observations. The physical properties of SCN and some input conditions used in simulation are summarized in Table 1 for reference.

The governing equations and their associated boundary conditions were solved numerically by an efficient finite-volume method scheme with a multigrid acceleration [8,9]. Second-order accuracy was also retained through the deferred correction. Detailed numerical implementation can be found elsewhere.

\section{Results and discussion}

Without rotation, the observed flow and interfaces from the laser light cut are shown in Fig. 2a; the exposure time was $15 \mathrm{~s}$. From this side view, as shown, there are two flow cells in the melt. The melt flows upwards at the center, while downwards near the interfaces. The upward melt (lighter) is also heated at the top wall and then flows to the two sides to melt back the interfaces. As a result, the upper part of the zone becomes wider. The downward melt (heavier) from the interfaces is warmed up again from the bottom wall at the center and then flows upwards. Although the picture shown in Fig. 2a looks like two-dimensional, the flow structure was fully three-dimensional. However, the flow patterns at other light-cut planes were much more difficult to observe. Increasing the heating power made the zone length longer, but the flow structure and the zone shape were not changed much. Also, the measured temperature at the lower part (between the heater and the ampoule) was slightly higher than the upper part; the lower part also melted first during zone formation. Therefore, the trapezoidal zone shape in Fig. 2a was dominated by the natural convection. This observation was also consistent with that reported by Pfann et al. [1,2]. Interestingly, if we imposed only $1 \mathrm{rpm}$ ampoule rotation, a significant change of the zone shape was observed, as shown in Fig. 2b; the exposure time was $11 \mathrm{~s}$. As shown, the zone shape becomes much more symmetric, and the upper and lower zone lengths are about the same. As compared with Fig. $2 \mathrm{a}$, the rotation reduced the upper zone length, while widened the lower part; the zone length seemed to be averaged by the rotation. The flow structure was also changed quite significantly. In fact, due to rotation, the flow visualization became much more difficult. The aluminum powder is heavier than the melt. As a result, larger particles tended to settle down, as shown by the bright area at the melt bottom in Fig. 2a. As the ampoule was 
Table 1

Physical properties and some input parameters [5]

\section{SCN}

Density of solid

Density of melt

Melting point

Heat of fusion

Thermal conductivity of solid

Thermal conductivity of melt

Specific heat of solid

Specific heat of melt

Thermal expansion coefficient

Pyrex tube

Density

Thermal conductivity

Specific heat

Surface emissivity

\section{Other input parameters}

Ampoule length for simulation

Crystal diameter

Ampoule diameter

Heater peak temperature

Far field heater temperature

Width of heating distribution

Peak heater temperature variation

Heat transfer coefficient

Rotation speed

Ampoule pulling speed

For the simplified model

Heating profile

$$
\begin{aligned}
& \rho_{\mathrm{c}}=\rho_{\mathrm{f}}=1.016 \mathrm{~g} \mathrm{~cm}^{-3} \\
& \rho_{\mathrm{m}}=0.988 \mathrm{~g} \mathrm{~cm}^{-3} \\
& T_{\mathrm{m}}=331.35 \mathrm{~K} \\
& \Delta H=46.5 \mathrm{~J} \mathrm{~g}^{-1} \\
& k_{\mathrm{c}}=k_{\mathrm{f}}=2.25 \times 10^{-3} \mathrm{~W} \mathrm{~cm}^{-1}{ }^{\circ} \mathrm{C}^{-1} \\
& k_{\mathrm{m}}=2.23 \times 10^{-3} \mathrm{~W} \mathrm{~cm}^{-1}{ }^{\circ} \mathrm{C}^{-1} \\
& C_{p_{\mathrm{c}}}=C_{p_{\mathrm{f}}}=1.955 \mathrm{~J} \mathrm{~g}^{-1}{ }^{\circ} \mathrm{C}^{-1} \\
& C_{p_{\mathrm{m}}}=2.0 \mathrm{~J} \mathrm{~g}^{-1}{ }^{\circ} \mathrm{C}^{-1} \\
& \beta_{\mathrm{T}}=8.1 \times 10^{-4} \mathrm{~K}^{-1}
\end{aligned}
$$

$$
\begin{aligned}
& \rho_{\mathrm{a}}=2.3 \mathrm{~g} \mathrm{~cm}^{-3} \\
& k_{\mathrm{a}}=0.014 \mathrm{~W} \mathrm{~cm}^{-1}{ }^{\circ} \mathrm{C}^{-1} \\
& C_{p_{\mathrm{a}}}=0.7535 \mathrm{~J} \mathrm{~g}^{-1}{ }^{\circ} \mathrm{C}^{-1} \\
& \varepsilon_{\mathrm{a}}=0.0
\end{aligned}
$$

$$
\begin{aligned}
& L=15 \mathrm{~cm} \\
& D_{\mathrm{c}}=0.9 \mathrm{~cm} \\
& D_{\mathrm{a}}=1.1 \mathrm{~cm} \\
& T_{\mathrm{p}}=111.85^{\circ} \mathrm{C} \\
& T_{\mathrm{a} \infty}=36.85{ }^{\circ} \mathrm{C} \\
& a_{\mathrm{f}}=a_{\mathrm{c}}=0.3 \mathrm{~cm}(0.257 \mathrm{~cm} \text { for Fig. } 5 \mathrm{~b}) \\
& \Delta T_{\mathrm{p}}=0.02 T_{\mathrm{m}} y / D_{\mathrm{a}}(y: \text { vertical distance from bottom }) \\
& h=1 \times 10^{-2} \mathrm{~W} \mathrm{~cm}{ }^{-2} \mathrm{C}^{-1} \\
& \Omega=0-1 \mathrm{rpm} \\
& U_{\mathrm{amp}}=0 \mathrm{~cm} / \mathrm{h} \\
& \\
& T_{\mathrm{a}}=76.85+50 \exp \left\{-[(z-0.5 L) / a]^{2}\right\}\left({ }^{\circ} \mathrm{C}\right) ; \\
& a=0.2 \mathrm{~cm} ; h=8 \times 10^{-3} \mathrm{~W} \mathrm{~cm}-2{ }^{\circ} \mathrm{C}^{-1}
\end{aligned}
$$

rotating, the settled powder was conveyed to the top and then resettled, and they were also visualized. As a result, some particle tracks near the heater seem to come across the flow lines of the main cells. Shortening the exposure time reduced these strange tracks. Nevertheless, one can still trace the flow lines near the interfaces to obtain a better picture. As shown, still, two flow cells remain. Such a significant change in the zone shape and the flow structure can be explained by three mechanisms: (1) forced convection due to rotation, (2) rotational convective heat transfer, and (3) the rotational melting $\left(\boldsymbol{n} \cdot \boldsymbol{e}_{\phi}>0\right.$ in the Stefan equation) and sol- idification $\left(\boldsymbol{n} \cdot \boldsymbol{e}_{\phi}<0\right)$, or one may call it the Stefan effect due to its role in Eq. (1). Pfann et al. [2] were the first to observe such a significant effect due to slow rotation, and similar nice photos for the zone shape (resorcinol at $2.5 \mathrm{rpm}$ ) were demonstrated, but without flow visualization. They used the convection mechanism and its coupling with the interfaces to explain such a dramatic change [1]: "the rising sheet of hot liquid can be regarded as a cutting or honing device which, combined with slow rotation, produces a near-planar interface much as the cutting blade of a lathe does to a round of metal." Such an explanation described correctly the 

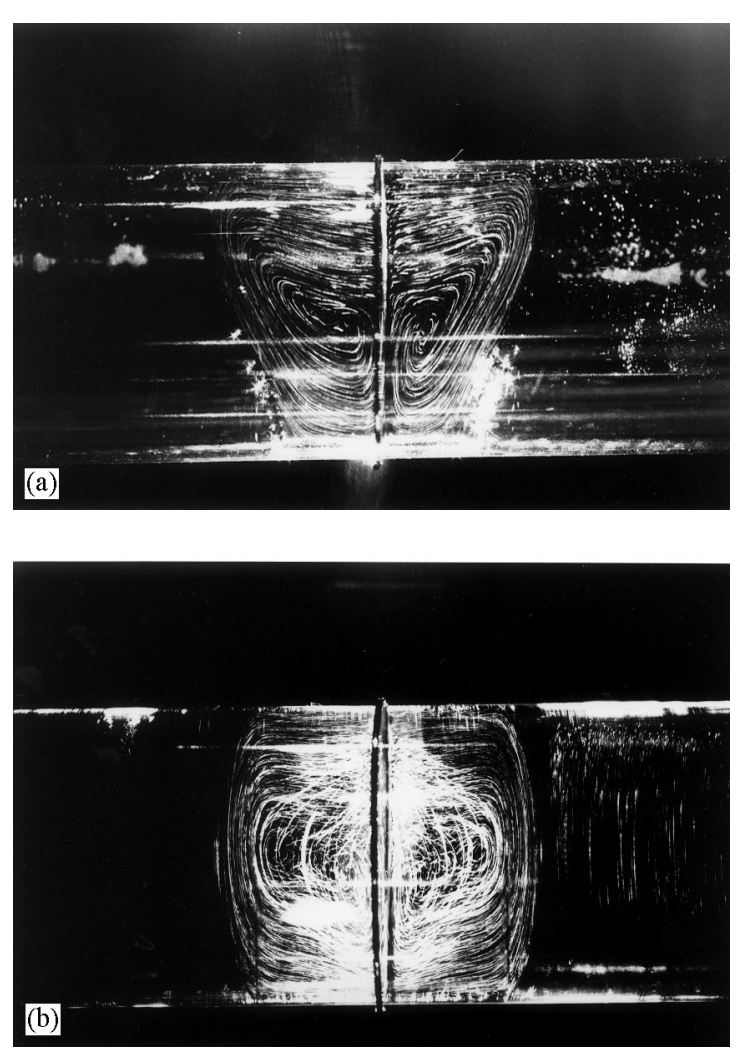

Fig. 2. Observed flow patterns and interface shapes: (a) $0 \mathrm{rpm}$ (exposure time was $15 \mathrm{~s}$ ); (b) $1 \mathrm{rpm}$ rotation (exposure time was $10 \mathrm{~s})$.

on-going process. However, the detailed interface control mechanisms are still not quite clear. For example, to control the zone shape effectively, the rotation cannot be too slow, while a too-low rotation speed is not a problem for metal cutting. Also, some part of the interfaces not only melted (abraded), but also grew. Furthermore, from Eq. (1), it is clear that the first two mechanisms will affect mainly the first two terms $\left(Q_{\mathrm{m}}\right.$ and $\left.Q_{\mathrm{s}}\right)$, while the third mechanism is due to the last term.

Before presenting the detailed simulation for Fig. 2, we illustrate first the heat flows by using a much simpler model. In this model, we only considered the melt and the interfaces were assumed to be flat. Because the interface shapes were fixed, Eq. (1) was not used. A similar ambient temperature distribution was used (see Table 1), while melting point at the interfaces was specified. A commercial package,
Fluent [4], was also used for comparison. As shown in Fig. 3, both for 0 and $1 \mathrm{rpm}$, our calculations were in good agreement with that by the Fluent code. For the case of no rotation, as shown on the left-hand side of Fig. 3a, similar to Fig. 2a, there are two main flow cells, and the isotherms are distorted significantly by the flows. Due to the convection, the isotherms near the interfaces at the top are much denser indicating the higher thermal gradients there (more effective heat transfer). The middle-cut of the flow and isotherms is shown on the right-hand side of Fig. 3a. The melt near the side wall flows upwards due to the higher temperature there; the major downward flows are near the interfaces, which cannot be seen from here. However, with $1 \mathrm{rpm}$ rotation, as shown in Fig. 3b, interestingly, both the flow structures and the isotherms are not changed much. This implies that the change of the interface shapes by the $1 \mathrm{rpm}$ rotation should be small as well, if the convection is the dominant mechanism. The main effect of the $1 \mathrm{rpm}$ rotation appears at the top of the zone. As shown on the right-hand side of Fig. 3b, the isotherms are distorted slightly in the rotational direction. The velocity vectors at the melt surface due to rotation can be clearly seen as well.

Therefore, from the calculations in Fig. 3, the fixed-interface model fails to provide an explanation for the rotational effect shown in Fig. 2. Nevertheless, from there we can conclude that the convection and the convective heat transfer enhanced by the $1 \mathrm{rpm}$ rotation are indeed not much. In other words, the first two terms in Eq. (1) were not much affected by the $1 \mathrm{rpm}$ rotation. On the contrary, if interfaces were taken into account, which required the use of Eq. (1) for determining the interface shapes, we were able to reproduce the observation in the experiments. As shown in Fig. 4, the calculations capture the key features of the experiments, both on the flow structures and interface shapes. For the case without rotation, as shown in Fig. 4a, the flow patterns and interface shapes are in good agreement with Fig. 2a. Due to the buoyancy force, the hotter (lighter) melt at the bottom floats upwards. It is warmed up again at the top wall, and flows to the two sides leading to a significant back melting of the interfaces at the top. The downward melt near the interfaces is 


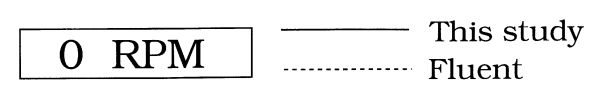

$$
\begin{aligned}
\Delta \mathrm{T}_{\max } & =31.92{ }^{\circ} \mathrm{C} \longrightarrow 0.1 \mathrm{~cm} / \mathrm{s} \\
\Delta \mathrm{T}_{\max } & =31.61{ }^{\circ} \mathrm{C}
\end{aligned}
$$
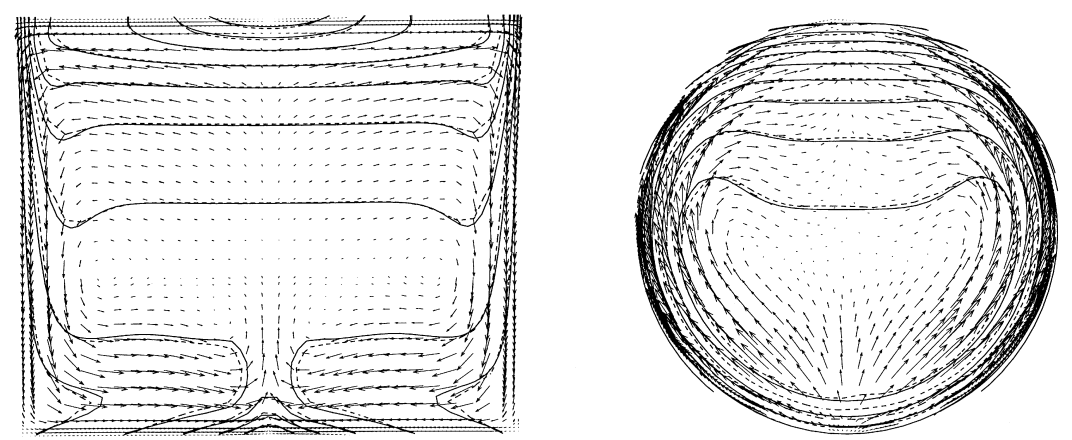

(a)
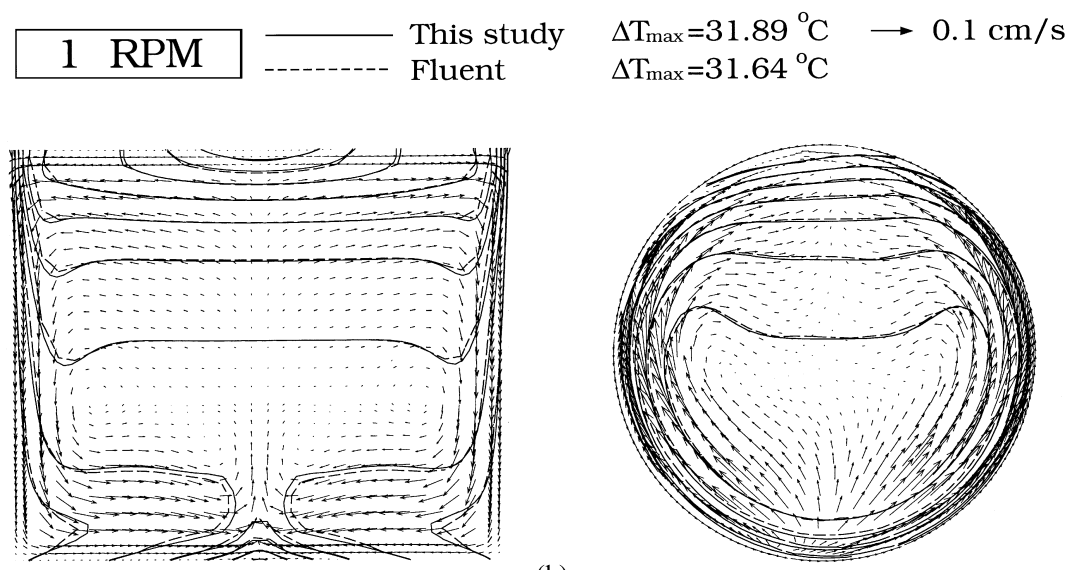

(b)

Fig. 3. Calculated flow patterns and isotherms for the simplified model: (a) $0 \mathrm{rpm}$; (b) $1 \mathrm{rpm}$. Dashed lines are the results obtained by using Fluent. The spacing for the isotherms is $\left(T_{\max }-T_{\mathrm{m}}\right) / 10$.

cooler and heavier, but it is heated up again by the heater at the bottom wall and floats up at the center. One can also get a better picture from the distorted isotherms, which are consistent with the flow directions. The other side view of Fig. $4 \mathrm{a}$ also illustrates the natural convection; the melt near the wall is heated and flows upward. One cannot see a downward flow from this plane, because the major downward flows appear near the interfaces. Also, due to the lens effect, we do not attempt to make an exact comparison here. In Fig. 2, the zone shape and the flow structures were stretched vertically due to the higher refractive indices of the melt and the ampoule. Nevertheless, the calculated up- per and lower zone lengths agrees well with the experiments within $5 \%$.

Interestingly, with $1 \mathrm{rpm}$ rotation, as shown in Fig. 4b, the zone shape becomes much more axisymmetric, which agrees well with the observation in Fig. 2b. The flow structure shown in Fig. 4b is also similar to that in Fig. 2b. One shall pay more attention on the flow shapes and vortices. Besides the agreement in the zone shape, it is interesting that the isotherms near the upper interfaces are still denser than the lower ones as that in Fig. 3a. The right-hand side of Fig. 4b also indicates that, though the effect of rotation becomes more obvious, the change of the flow and isotherms is not too 


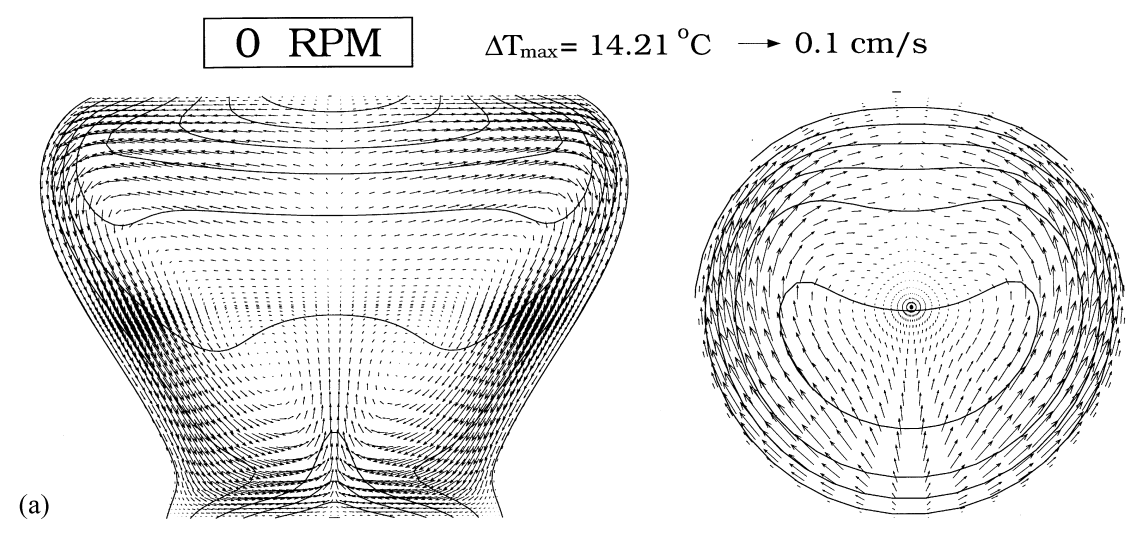

(b)
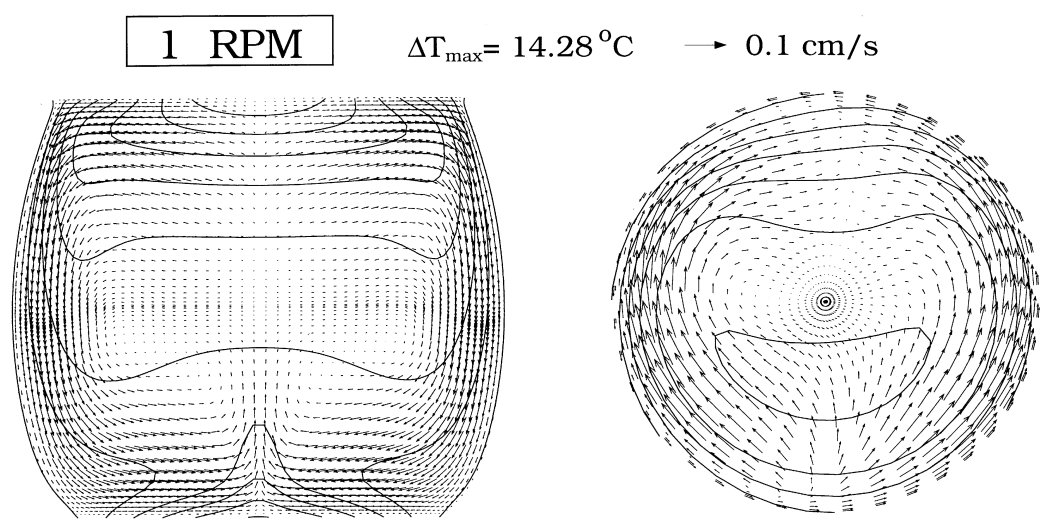

$1 \quad \mathrm{RPM} ; \mathrm{St}=0 \quad \Delta \mathrm{T}_{\max }=15.25^{\circ} \mathrm{C} \rightarrow 0.1 \mathrm{~cm} / \mathrm{s}$

(c)
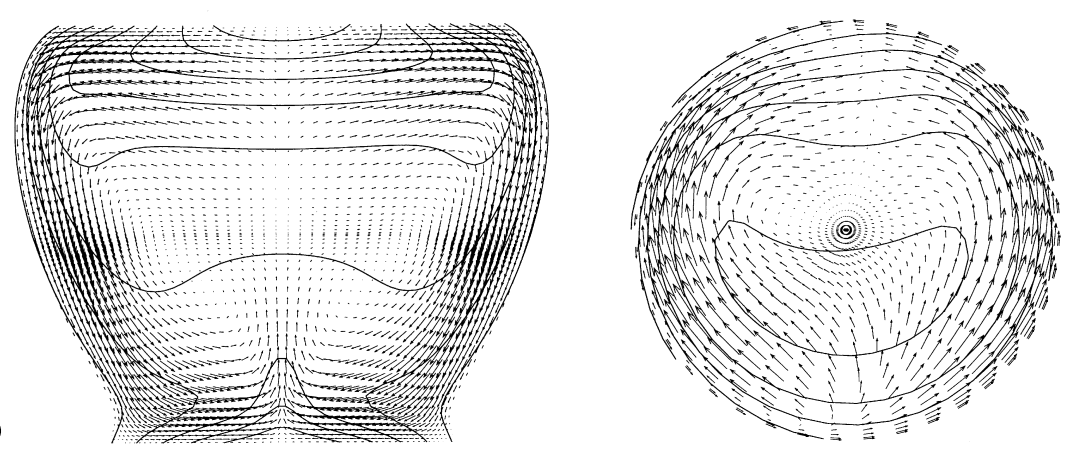

Fig. 4. Calculated flow patterns, isotherms, and interface shapes for the full model: (a) $0 \mathrm{rpm}$; (b) $1 \mathrm{rpm}$; (c) St = 0 at $1 \mathrm{rpm}$. The spacing for the isotherms is $\left(T_{\max }-T_{\mathrm{m}}\right) / 10$.

significant. Indeed, the success of the full-model calculation in Fig. $4 \mathrm{~b}$ is due to the consideration of the interface energy balance. As shown in Eq. (1), for steady rotation without ampoule translation $\left(\partial h_{\mathrm{i}} / \partial \tau=v_{z}=0\right), r \Omega\left(\boldsymbol{e}_{\phi} \cdot \boldsymbol{n}\right)$ plays a crucial role. With rotation, at the interfaces continuous solidification occurs at $\boldsymbol{e}_{\phi} \cdot \boldsymbol{n}<0$, while melting at $\boldsymbol{e}_{\phi} \cdot \boldsymbol{n}>0$. For the left interface, at the front side, 
$\boldsymbol{e}_{\phi} \cdot \boldsymbol{n}<0$ and the solidification dominates. The heat of fusion is released to the melt and it distorts the interface towards the rotational direction. Meanwhile, the melting occurs at the back side, and the heat of fusion is extracted from the melt making the solid interface intrude towards the melt in the rotational direction there. As a result, the zone length is averaged out with the period of rotation. This mechanism (the Stefan effect) is dominant for the interface shapes here and cannot be ignored in the simulation.

To better illustrate the significance of this Stefan equation, we also performed a calculation by purposely letting $\Delta H=0$ (or $\mathrm{St}=0$ ); the last term of Eq. (1) was removed. Fig. 4c shows the calculated result. As shown, it fails to reproduce the observation in Fig. 2b. The calculated interface shapes are about the same as that in Fig. 4a. As expected from Fig. 3, the effect of $1 \mathrm{rpm}$ rotation without the heat of fusion is indeed not much. Therefore, from these calculations, it is clear that the heat of fusion (or the Stefan effect) plays a crucial role in the Pfann-Miller technique. In fact, Pfann et al. [1,2] at their time might be aware of this effect, but the continuous rotational solidification and melting was not discussed. Their explanation using the "cutting blade" of the natural convection during rotation was right (at least for melting), but it was a little vague. We also performed calculations for zero gravity (without natural convection) with a highly asymmetric heating, a nearly axisymmetric zone shape was obtained as well. Therefore, the natural convection is not crucial to the axisymmetric zone. Nevertheless, if a very short zone is preferred, any method of enhanced flows (e.g., natural convection or the accelerated crucible rotation technique (ACRT) [10]) could be helpful. Of course, using the natural convection is the easiest. For most high-Prandtl-number materials, the Stefan effect can be significant even at slow rotation because the heat of fusion released or absorbed is more difficult to be removed due to the poorer thermal conduction. On the other hand, for lower-Prandtl-number materials, such as semiconductors or metals, the Stefan effect can also be significant, but it requires a much higher rotation speed. Meanwhile, in that case, the role of convective heat transfer in the rotational direction becomes more important.
The control of a flat growth interface, which is preferred for single-crystal growth, by the Pfann-Miller technique [2] was also quite straightforward. As the heating power was reduced, the zone length was reduced. Meanwhile, the interfaces became much flatter as shown in Fig. 5a. Final calculation was made for this situation. The results are shown in Fig. 5b. As shown, the flow structures and isotherms are still similar to the previous calculations in Fig. 4b, but the interfaces are much flatter. Of course, the superheating of the melt is lower. Since a very short zone with flat interfaces can be controlled easily, the technique is popular in zone refining, especially for organic compounds.

Even though the Pfann-Miller technique has been proven to be a useful process for HZM, there is a side effect due to the rotation that has not yet been discussed. Similar to the Stefan effect, the rotational segregation [11] can be significant as well depending on the rotation speed. In certain more severe growth conditions, the rotational constitutional supercooling may be of significance. To illustrate that, we purposely used the less-pure materials (left over from the zone refining) to do the zone melting, and a higher growth rate was used $(2 \mathrm{~cm} / \mathrm{h})$. The zone length was kept the same as that in Fig. 2. The crystal grown without rotation remained transparent and clear. However, at $1 \mathrm{rpm}$ rotation, some bands of impurities were found, as shown on the left-hand side of Fig. 6. The shape of the bands was consistent with the observed growth interface. Interestingly, as the rotation speed was increased to $3 \mathrm{rpm}$, clear inclusions (bubbles) started to appear as shown in Fig. 6, and finally the core of the grown crystal became opaque. The macroscopic banding of the inclusions with a lower frequency may be due to the periodic breakdown of the growth front. We have also carried out simulation for higher rotation speeds, but have not found any unstable flows. Making the growth interface flatter reduced the inclusions significantly. Particle inclusions by rotation were also discussed by Wilcox et al. $[12,13]$, but they found that the amount of inclusions seemed to decrease with the increasing rotation speed. Because the rotation speed they used was much higher (ranging from 5 to $35 \mathrm{rpm}$ ) and the 


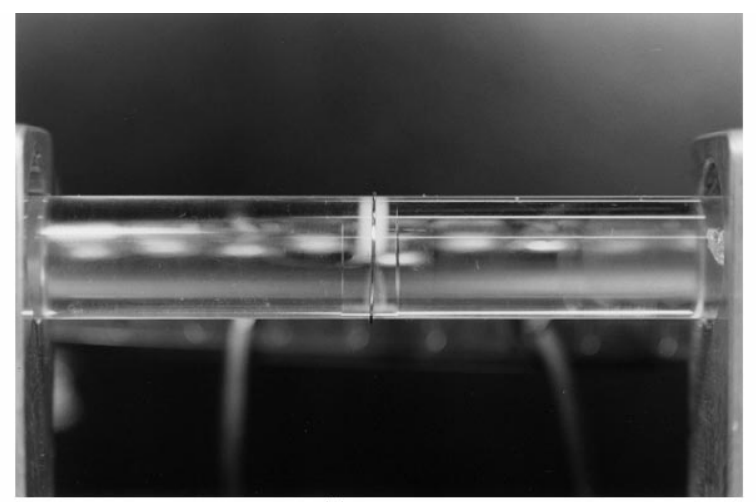

(a)

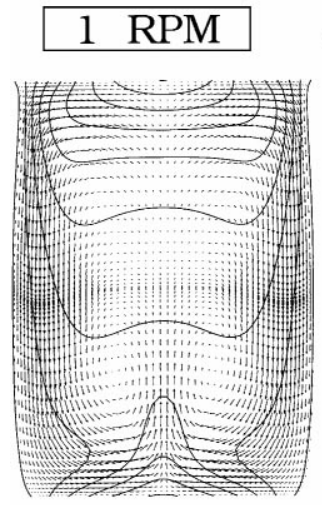

$$
\Delta \mathrm{T}_{\max }=10.57^{\circ} \mathrm{C} \quad \rightarrow 0.1 \mathrm{~cm} / \mathrm{s}
$$

(b)

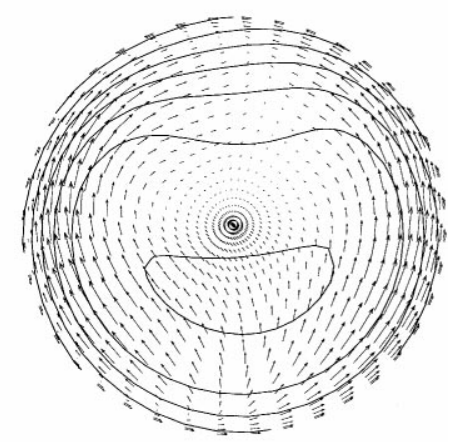

Fig. 5. (a) Observed short zone and flat interfaces; (b) calculated isotherms, flow patterns, and interface shapes; the spacing for the isotherms is $\left(T_{\max }-T_{\mathrm{m}}\right) / 10$.

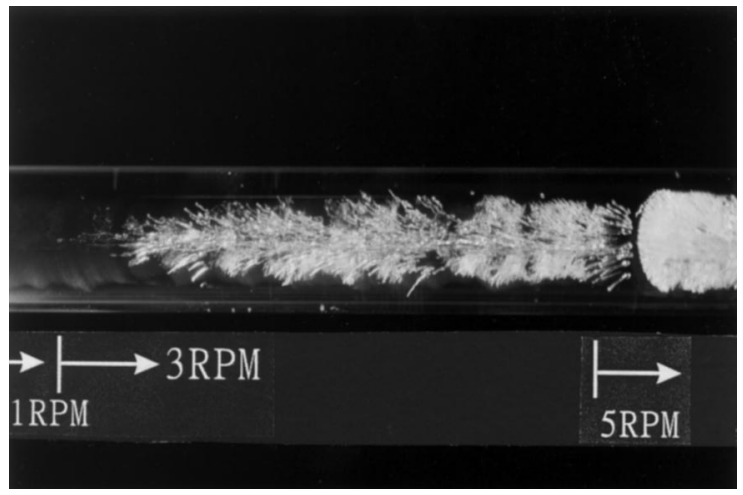

Fig. 6. Appearance of the grown crystal at 1 and $3 \mathrm{rpm}$ ampoule rotation.

inclusions were solid particle (size ranging from 10 to $100 \mu \mathrm{m})$, their inclusion mechanisms could be different.

\section{Conclusions}

Through the visualization and computer simulation of the flows and interfaces during the horizontal zone-melting of SCN, we provided a clear picture for the interface control mechanisms by slow rotation. It was found that the heat of fusion, which was released or absorbed at different places of the interfaces during rotation (the Stefan effect), played a crucial role. Without rotation, significant thermal asymmetry was caused by natural convection leading to a highly nonuniform zone with a trapezoidal shape at its side view. With $1 \mathrm{rpm}$ rotation, the asymmetric thermal field caused by the natural convection was not altered much. However, some part of the interfaces remelted (was abraded) and extracted the heat of fusion from the melt, while some part of the interfaces regrew and 
released the heat of fusion to the melt. This process continued with the period of the rotation and averaged out for the interfaces leading to a more axisymmetric zone. If the interfaces had no resistance to the heat flow (without the heat of fusion), the change of the trapezoidal zone shape by the $1 \mathrm{rpm}$ rotation was small. For high-Prandlt number materials, such as organic compounds or oxides, this Stefan effect could be significant even at slow rotation. Apparently, for materials with a much lower Prandtl number, such as semiconductors or metals, or lower heat of fusion, a much higher rotation speed is required to have a comparable effect. Besides the heat transfer, the rotational remelting and regrowth might have significant influences on segregation and constitutional supercooling. Although the evidence seems to be clear here, further study is necessary for better understanding.

\section{Acknowledgements}

The authors are grateful for the constructive comments from the referee. Some useful references provided by Prof. W.R. Wilcox are highly appreciated. This work was sponsored by the National
Science Council and the National Center for High Performance Computing of the Republic of China under Grant No. NSC89-2214-E002-040.

\section{References}

[1] W.G. Pfann, Zone Melting, 2nd edition, Wiley, New York, 1966, p. 97.

[2] W.G. Pfann, C.E. Miller, J.D. Hunt, Rev. Sci. Instrum. 37 (5) (1966) 649

[3] W.R. Wilcox, R. Friedenberg, N. Back, Chem. Rev. 64 (1964) 187.

[4] Fluent UNS 5.0 Usual Manual, Fluent Inc., 1999.

[5] M. Yao, H. de Groh III, Numer. Heat Transfer, Paper A 24 (1993) 393.

[6] E. Rubinstein, M.E. Glickman, B.W. Mangum, Q.T. Fang, N.B. Singh, J. Crystal Growth 89 (1988) 101.

[7] C.W. Lan, M.C. Liang, J. Crystal Growth 208 (2000) 327.

[8] M.C. Liang, C.W. Lan, J. Comput. Phys. 127 (1996) 330.

[9] C.W. Lan, M.C. Liang, J. Comput. Phys. 152 (1999) 55.

[10] H.J. Scheel, J. Crystal Growth 13/14 (1971) 560.

[11] C.W. Lan, M.C. Liang, J.H. Chian, J. Crystal Growth 212 (2000) 340.

[12] R.B. Fedich, W.R. Wilcox, Sep. Sci. Technol. 13 (1) (1980) 31.

[13] J.E. Coon, W.R. Wilcox, Sep. Sci. Technol. 15 (7) (1980) 1401. 